\title{
Emerging Chinese-English Hybridized Internet Neologisms: a Big Data Study Based on Baidu Index
}

\author{
Huayong Li \\ Foreign Language School, Sichuan University of Arts and Science, Sichuan Province, People's Republic of China \\ E-mail: lihuayong2008@126.com
}

$\begin{array}{lc}\text { Received: January 16, } 2020 & \text { Accepted: February 20, } 2020 \quad \text { Online Published: March 7, } 2020 \\ \text { doi:10.11114/ijecs.v3i1.4688 } & \text { URL: https://doi.org/10.11114/ijecs.v3i1.4688 }\end{array}$

Abstract
This article studies the emerging neologisms in the Chinese cyber context: 打 call, hold 住, word 哥, which are termed
Chinese-English hybridized internet neologisms (CEHINs). CEHINs share the features of Chinese words and English
words. Based on the research tool called Baidu Index, it is found: (1) the structure and pronunciation of CEHINs ( $f J$
call, hold 住, word 哥) is different from the 'pure' Chinese or English internet neologisms; (2) the diffusion of them is
Chinese netizen-driven, not the meme-driven; (3) the top users of them are usually aged between 30-39, while the 'pure'
Chinese internet neologisms are much more frequently used by netizens aged 20-29; and (4) the popularization of
internet, fast cultural communications in cyber times, and the further pervasiveness of English as a Lingua Franca in
China are the reasons for the emergence and popularity of them in China.

Keywords: Neologism, English word, Chinese character, cyber context

\section{Introduction}

With the popularization of the internet, and the pervasiveness of English in China, a new type of internet neologism is emerging in recent years: such as 打 call (pinyin: $d a$ call, 'cheer for'), hold 住(pinyin: hold zhu, 'control'), word 哥 (pinyin: word ge, 'amazing', 'my brother'). These neologisms are formed by a Chinese character plus an English word, or an English word plus a Chinese character. Hence, the author terms them Chinese-English hybridized internet neologism (CEHIN). They are frequently and widely used by Chinese netizens: 打 call is the top one annual Chinese internet neologism in 2017, hold 住 is the top one annual Chinese internet neologism in 2011, and word 哥 is the ninth top annual Chinese internet neologism in 2016, according to the report from official weibo ${ }^{i}$ of the journal yaowen jiaozi. Through the lens of internet neologisms we can observe the behaviour of netizens, the thought change of the netizens, the internet culture change and even the Zeitgeist of an era so that the internet neologisms have attracted the eyes of the scholars (Roigmarín, 2016; Zhou \& Shu, 2017; Zhang, 2017; Xu \& Tian, 2017).

However, the scholars have not paid much attention to the emerging CEHINs. One reason may be that they are emerging and used in recent years: 打 call ( initially in May, 2017), word 哥(initially in August, 2016), hold 住(initially in September, 2011). These neologisms are worth being studied in details for the following reasons: (1) they show the blending of English words and Chinese characters in cyber times in China; (2) their structure is different from the typical 'pure' Chinese or English internet neologisms that are completely formed by Chinese character or English letters; (3) they show the creativity and ways of thinking of Chinese netizens; and (4) they are frequently and widely used by Chinese netizens.

There is a trend toward internet neologism research with the empirically reliable methods, such as corpus linguistic method, big data or quantitative linguistic method. The data-based method is helpful to reveal the authentic using status and the intrinsic characteristics of the neologisms, discovering what could not be noticed previously or what could not be studied by the traditional methods. Consequently, the author conducts the research to 打 call, hold 住, word 哥, based on the big data retrieval tool called Baidu Index. ${ }^{\text {ii }}$

\section{Research Questions}

Based on the Baidu Index, the author tries to answer the following three questions related to the CEHINs 打 call, hold 住, word 哥: (1) what are the features of them? (2) why are they emerging? and (3) what are the prospects for them? 


\section{Research Methodology}

\subsection{Research Tool}

The author is based on Baidu Index in this research. It is developed by Baidu, Inc., the second biggest search engine company in the world. Baidu search engine holds $76.05 \%$ market shares in China's search engine market for 0.751 billions netizens in 2017, according to The $40^{\text {th }}$ China Statistical Report on Internet Development. ${ }^{\text {iii }}$ Baidu Index is regarded as one of the most important analysis tools in the internet times and even the data times ${ }^{\text {iv }}$ so that it can relatively much more accurately reveal or represent the authentic behaviours and needs of Chinese netizens. Baidu Index, which is based on the tremendous big data of Baidu search engine, not only analyse the 'search heat' degrees of the keywords but also mine the public opinion information, the market demands, the user features, and so on. ${ }^{v}$ It is updated everyday and provides the data and analysis on the pc net and mobile net. For this research, Baidu Index can quickly and automatically provide the following retrieval information related to the three neologisms 打 call, hold 住, word 哥: the users, the behaviors of netizens, the diachronic and synchronic frequency, the gender and age of netizens, the distributional areas, and so on, which can substantially answer the previous research questions. Therefore, Baidu Index can be viewed as a tremendous big data based research tool and market demand analysis tool.

There are advantages of big data based method: it can reflect some intrinsic features of languages, one of which is the probability of language (Bod, et al., 2003); it can also reveal more language facts, usage patterns, the diachronic trend, the nature of language and so on, which are impossible to find under the small linguistic data, or introspection data. Consequently, based on Baidu Index, the paper tries to observe and analyse the origin, the diachronic and synchronic features of the neologisms 打 call, hold 住, word 哥, which are helpful to study the features of the emerging CEHINs.

\subsection{Object of Research}

The research object is the three CEHINs 打 call, hold 住, word 哥, focusing on the features, reasons of emergence, and the prospects of them. The reasons for selecting the three neologisms are: (1) they are respectively listed as one of the annual top ten Chinese internet neologisms in 2017 (打 call), 2016 (word 哥), 2011 (hold 住), which is released by the official weibo of the Chinese journal called yaowen jiaozi; (2) they are typical CEHINs; (3) they are frequently and widely used by Chinese netizens during cyber communications; and (4) they emerged in different years, which is helpful for the diachronic and synchronic study to CEHINs.

\subsection{Procedures}

The author mainly conducts this research based on Baidu Index in the following steps:

(1) Analyse the structure and semantic features of the three neologisms 打 call, hold 住, word 哥. In order to study the structure and semantic features of CEHINs, the author analyses the components of the three neologisms based on the word frequency from the List of Lexicon of Common Words Contemporary Chinese (draft) (the Lexicon of Common Words Contemporary Chinese Project Team, 2008) and the corpus created by Mark Davies. ${ }^{\mathrm{vi}}$

(2) Analyse the using features of CEHINs. The author retrieves the neologism fJ call on Baidu Index, focusing on initial searching status, user's distribution in cities, user's age and gender distribution, which can be automatically and objectively obtained in the 'search trend' function and the 'user's portrait' function of Baidu Index ${ }^{\text {vii }}$. In order to observe the life stage of CEHINs, the author also retrieves hold 住, word 哥 in Baidu Index. Based on the above retrieval results, the using features of CEHINs can be summarized and explained.

(3) Analyse the reasons for the emergence of CEHINs. Based on the retrieval results from Baidu Index and other websites, the author analyses the reasons why the CEHINs are emerging and popular.

(4) Analyse the prospects for CEHINs.

\section{Features of CEHINs}

\subsection{Structure and Semantic Features of CEHINs}

The emerging CEHINs, such as 打 call, hold 住, word 哥, 太 out, 很low, take on the four structure features. (1) The component words of them are single syllable. Regardless of English words or Chinese characters in the neologisms, they are all single syllable, such as 打 /dă/, or call /ks:l/in the neologism of fJ call. (2) The component words of them is high frequency words. For example, Chinese characters 打 (409) viii,, 住(250), 太 (237), 很 (40) are within the top 500 out of 56008 Chinese words, according to the List of Lexicon of Common Words Contemporary Chinese (draft) (the Lexicon of Common Words Contemporary Chinese Project Team, 2008). Obviously, the English words, call, hold, out, low are within the top 300 high frequency words: call (122) ix $^{\mathrm{x}}$ hold (213), word (244). (3) The number of letters of English word or strokes of the Chinese character in 打 call, hold 住, word 哥 is less: letters of the English word are no more than four, and the strokes of the Chinese character are no more than 9. The less letters or strokes in the hybridized internet neologisms conform to the economy principle of language, which is convenient for the use and diffusion of the 
neologisms in Chinese cyberspace communication context. (4) They show the features of English and Chinese in pronunciation and meanings, containing two different kinds of phonetic sounds: for example, in the neologism 打 call, the first part 打 is pronounced /dă/ in pinyin of Chinese phonetic system while the second part call is pronounced /ko:1/ in English phonetic system; the meaning of 打 call is together produced by the components of the Chinese character fJ and the English word call.

The meaning of Chinese-English hybridized internet neologisms gives the audience or users a sense of humor, fashion, or positive feeling when they are used in cyberspace communications in China. The three neologisms possess positive connotation and usually are used to express or construct positive semantic prosody in the cyberspace communications. Hence, they are frequently and widely used in Chinese netizens, which can be showed in the top ten annual Chinese internet neologisms in the passing decade: 打 call is top one in 2017; hold 住 is top one in 2011; word 哥 is the ninth top annual Chinese internet neologism in 2016.

\subsection{Using Features of CEHINs}

Firstly, they usually spread from 'the bottom to the up' that means they are initially used in the informal registers, and then in the much formal registers, even enteringthe quite formal register such as the mainstream media. They can truly reflect the conditions and the needs of majority common individuals: the netizens in China. The CEHINs are all initially used by Chinese netizens, especially in their cyber communications. With the increase of using frequency, they may spread out to much more formal registers, such as newspapers and TV programs, classrooms, the journals, and even the Chinese government websites. There is some evidence for this claim: (1) in CCTV (www.cctiv.com.cn) and China daily (www.people.com.cn), a great number of concordances on the three neologisms can be retrieved; (2) it can be seen that the neologisms 打 call, hold 住, word 哥, are also used in the classrooms by the teachers or students, and in the speeches of the much formal meetings; (3) the three neologisms have appeared in the China journals ${ }^{\mathrm{x}}$; and (4) in the quite official and formal website The Central People's Government of the People's Republic of China (www.gov.cn), some of the concordances on the three neologisms can also be retrieved, which suggests that these neologisms are accepted by the upper class or the elites.

Secondly, the initial diffusion of them is not based on the traditional so-called meme-driven. For example, when the hybridized neologism fJ call was coined in the internet, it was not diffused by 'meme'. Because, initially, the Chinese netizens want to know the meaning and implicature of fJ call, it is impossible for them to copy, simulate and use it directly. The memetic theoretical explanation to neologisms considers the neologism to be diffused by meme at the whole life cycle, including at the initial stage. The author retrieves the fJ call in Baidu Index, focusing on the 'needs of searching users' of the first month. The retrieval steps are: (1) put the neologism fJ call into the Baidu Index 'search box' and search; and (2) in searching result page, click the 'demand net' button in the menu, and then Figure 1 is automatically generated. According to Figure1 it can be found that Baidu Index retrieval results of the hybridized internet neologism 打 call are against the memetic theoretical explanation to initial diffusion of neologisms: 打 call is initially searched for the meaning and the Chinese netizens use it, which means that it is not directly used by the netizens at the initially stage, while it is supposed to be used directly according to the memetic theory. From Figure 1, it can be seen that within the first month, almost all the user's searching of fJ call is related to the meaning of fJ call, the action of 打 call, the course of 打 call, which are just to know the meaning and implicature of 打 call. After getting the background information of 打 call, Chinese netizens gradually use it in their cyber communications. So the author states that the initial diffusion of the Chinese-English internet hybridized internet neologisms 打 call, hold 住, or word 哥, are netizen's need-driven, not the meme-driven.

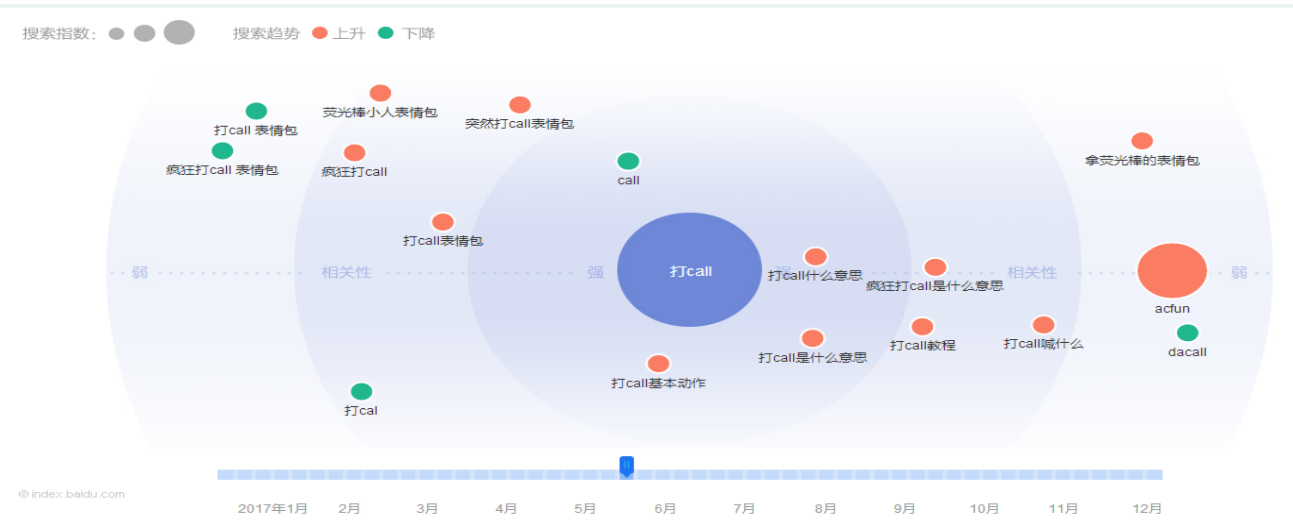

Figure 1. The first month searching for 打 call 
Thirdly, the using features of them are netizen-driven. Liu \& Lin (2018) argue that language is a complex adaptive system driven by human. The neologism is a sub-system of the complex adaptive system. Hence, the author may deduce that the using features of internet neologism are netizen-driven. The author retrieves the distribution of fJ call in Baidu Index: (1) put the neologism 打 call into the Baidu Index 'search box' and search; (2) in searching result page, click the 'user's portrait' button in the menu; (3) and then click the 'city' button in the 'district distribution' section; and (4) after that, Figure 2 can be automatically generated. It is found that Baidu Index trend of CEHIN fJ call shows the obvious netizen-driven characteristics: the more Chinese netizens are in a district, the more frequencies of the word fJ call in this district; there are several 'using centres' for the neologism of fJ call. The top-three 'using centres' for the neologism of fJ call are Beijing, Shanghai, Guangzhou, where according to the China Statistical Report on Internet Development ${ }^{\mathrm{xi}}$, the popularization rate of netizens are the top three in China.
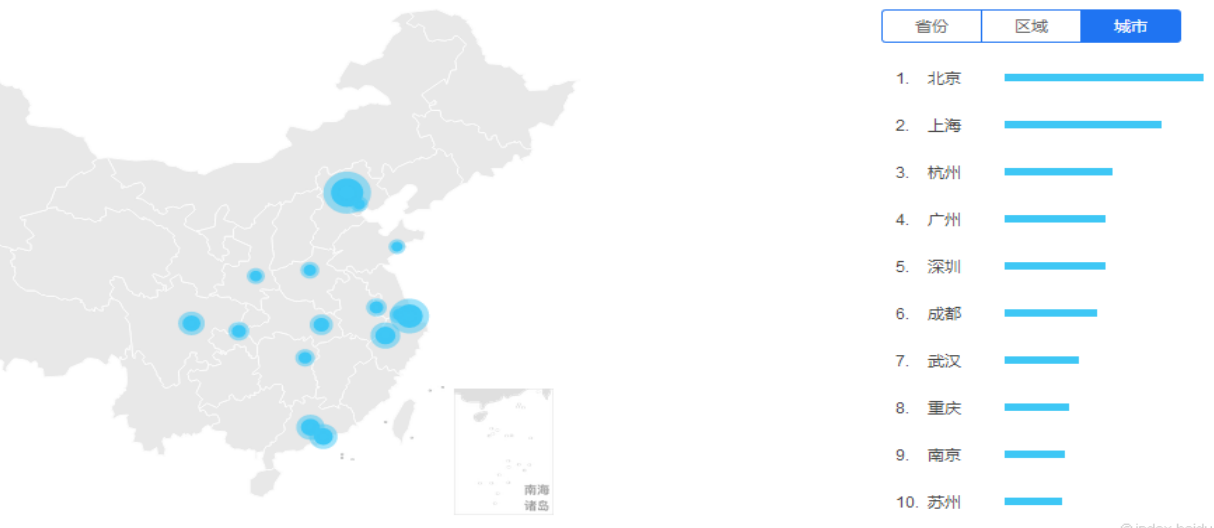

Figure 2. 'Using centres' of 打 call in China

Fourthly, there is region limitation for the popularity of Chinese-English hybridized internet neologisms. Based on the Baidu Index retrieval results of the three neologisms, the author finds the CEHINs which are popularly used in Chinese mainland are not used in Hong Kong, Taiwan, and Macao. Though the internet can eliminate the barricades of space and geography, and the communications in the internet are quite convenient and easy, the Chinese-English hybridized internet neologisms 打 call, hold 住, word 哥, are not used by the netizens in Hongkong, Taiwan, and Macao.

Finally, the age and gender distribution of users of 打 call, hold 住, word 哥 are quite different from users of other 'pure Chinese' internet neologisms, such as 图样图森破 (pinyin: tuyang tusen po, 'naive'). In order to observe the difference, the author retrieves the age and gender distribution of the neologism 打 call: (1) put the neologism fJ call into the Baidu Index 'search box'; (2) search and then click the 'user's portrait' button; and (3) Figure 3 can be automatically generated. Likewise, Figure 4 can be easily generated, which is used to visualize the age and gender difference between CEHINs and the 'pure' Chinese internet neologisms. From Figure 3, it can be found: (1) the top one group users of 打 call are aged between 30-39 years old, which accounts for 53\%; (2) the top two group users are aged between 40-49 years old, which accounts for $25 \%$; and (3) the proportion of male users of 打 call is $52 \%$, and the female is $48 \%$, which means that the male users almost are equal to the female users, and there is no gender difference in using the hybridized neologism 打 call. Dissimilarly, from Figure 4 it can be found that the users of the 'pure' Chinese internet neologism 图样图森破 are different: the top one group users are aged between 20-29 years old, which account for $37 \%$, while the top two group users are aged between 30-39 years old, accounting for 33\%; the proportion of male users are $78 \%$ and the female users are $22 \%$. The proportion of male users of 图样图森破 is obviously larger than that of female users: surpassing $56 \%$, which means that there is palpable gender difference in using the 'pure' Chinese internet neologism 图样图森破. More details of age difference in using the Hybridized and the 'pure' neologisms can be seen in Figure 3 and Figure 4. 


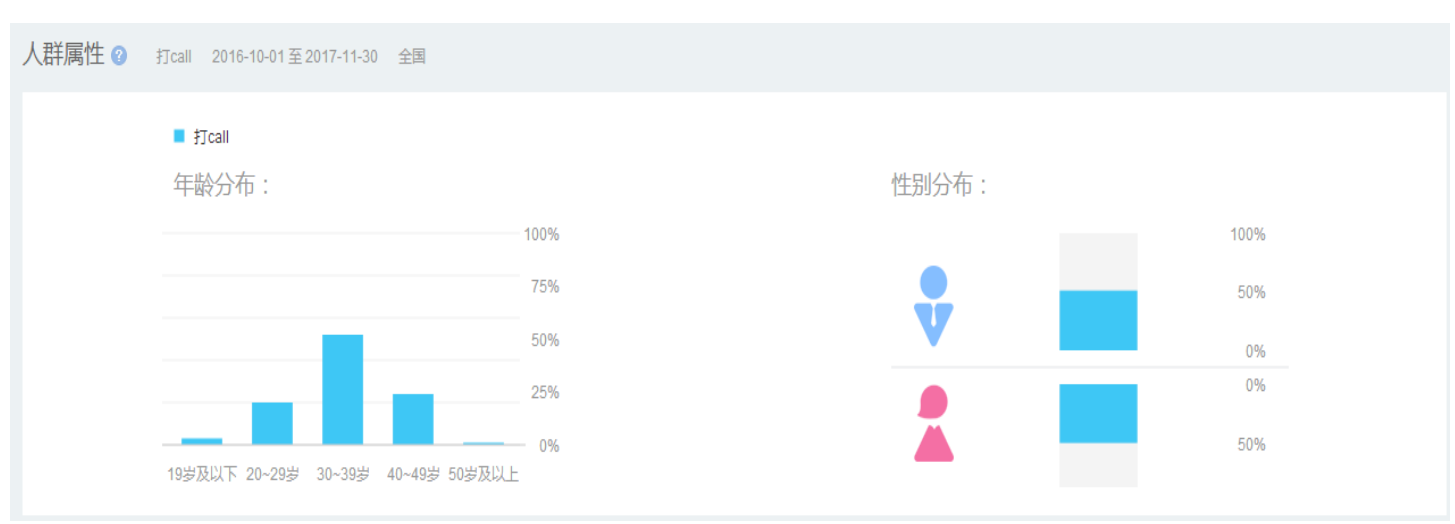

Figure 3.The age and gender distribution of 打 call

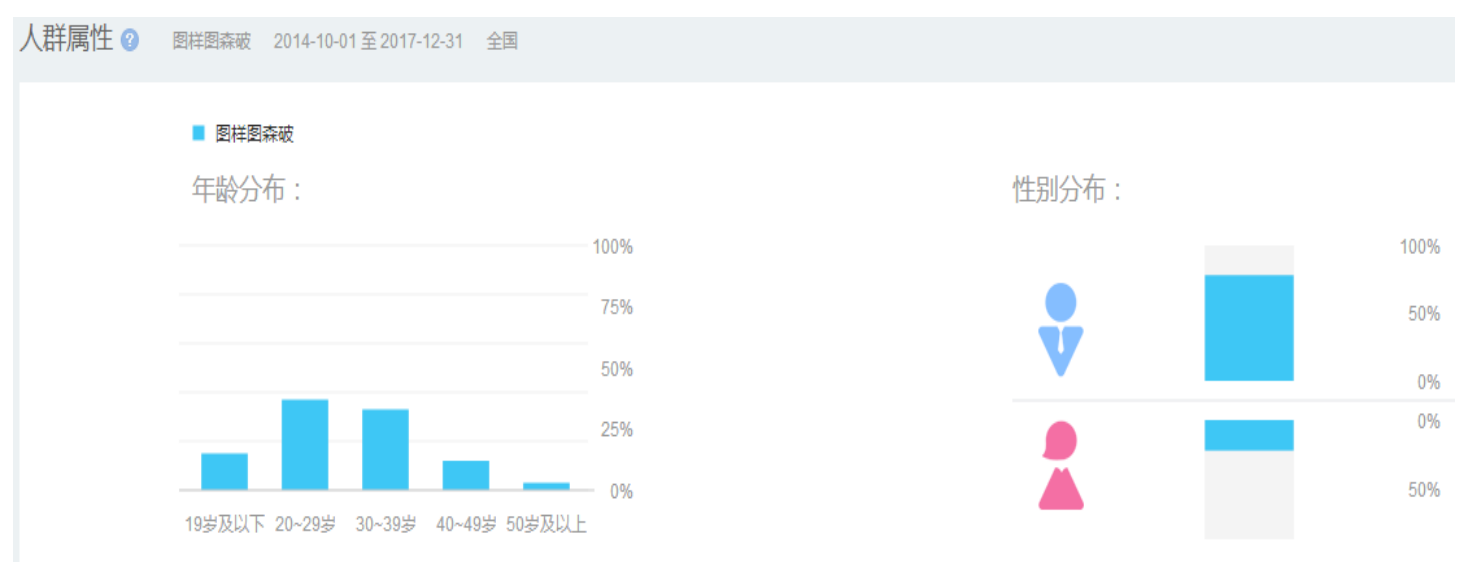

Figure 4. The age and gender distribution of 图样图森破

\subsection{Life Stage of CEHINs}

The CEHINs usually reaches the peak within short time because of certain events, and goes down quickly, and then keeps stable. Scholars have studied the life stage of neologisms. Based on the structural, social-pragmatic, and cognitive factors, Schmid (2008) put forward three stages: creation, consolidation, and establishing. Quantitative research on neologisms has also made great progress in statistical understanding of neologism life-stage: originated, evolved, die out (Zhuo \& Shu, 2018). Quantitative research views the word frequency as a leading factor in explaining life-stage and the prediction force of whether a neologism may survive or not. The frequency is significant to observe and understand the evolution features of CEHINs. The author retrieves the diachronic process of the neologism fJ call: (1) put the neologism 打 call into the 'search box' of Baidu Index; (2) click the button 'all' in the 'whole trend' section; and (3) Figure 5 can be automatically generated. In the same steps, Figure 6 and Figure 7 can be automatically generated. Figure 5 shows the origin, increasing, peak and decreasing of 打 call. There are no more than two months for fJ call to

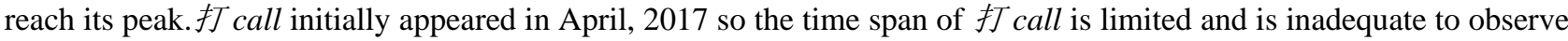
or describe the life stage of CEHINs. The author retrieves another neologism hold 住 which appeared in August, 2011, see Figure 6 which shows that the neologism hold 住 was suddenly popular among the Chinese netizens, in August, 2011 because of the Taiwan TV program called 大学生了没xii (Pinyin:'da xuesheng le mei') on 9th August, 2011. Within a week (from 21th August, 2011 to 28th August, 2011), the frequency of neologism hold 住 reached its peak (40,000 hits) and then dramatically decreased, and after 11th November, 2011, it kept stable. From 2012 to 2017, the frequency of hold 住 is relatively stable, 251 times per week. Similarly, there is also a peak for the neologism word 哥, and then goes down quickly, see Figure 7.

Based on Figure 6 and Figure 7, it can be seen that the diachronic tendency of hold 住 and word 哥 is quite similar. They are continually used by the Chinese netizens and gradually diffuses in other registers, such newspaper, TV program, and so on. Diachronically speaking, the CEHINs 打 call, hold 住, word 哥 are welcomed by the Chinese netizens because of theirs structure features and humorous semantic, which are elaborated in previous 'structure and semantic features of CEHINs' section. 


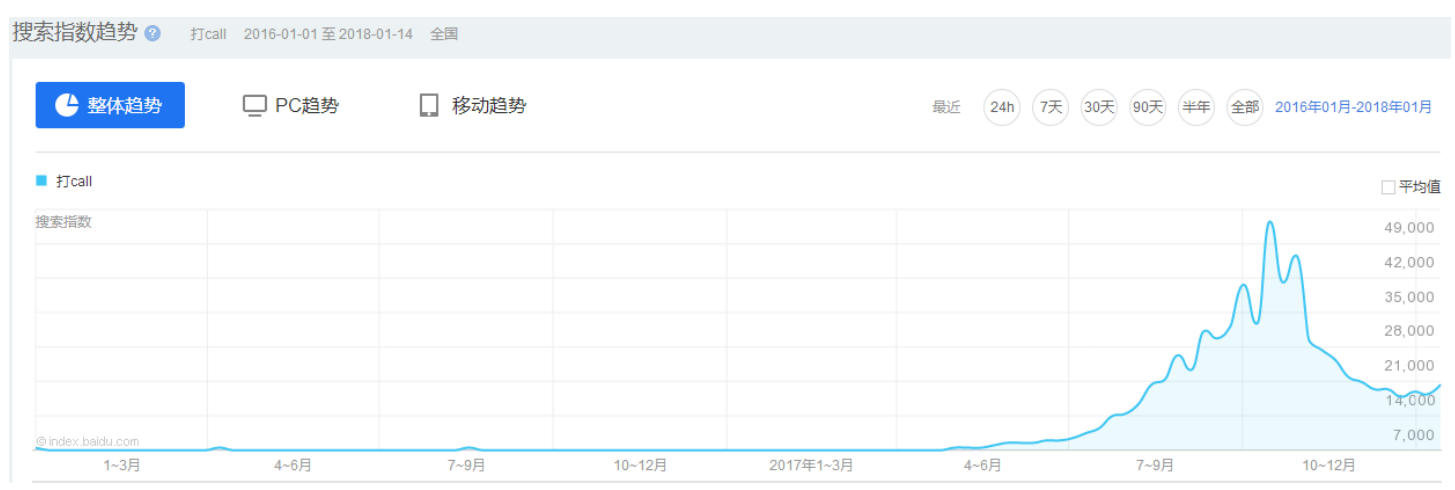

Figure 5. The diachronic using frequency of 打 call

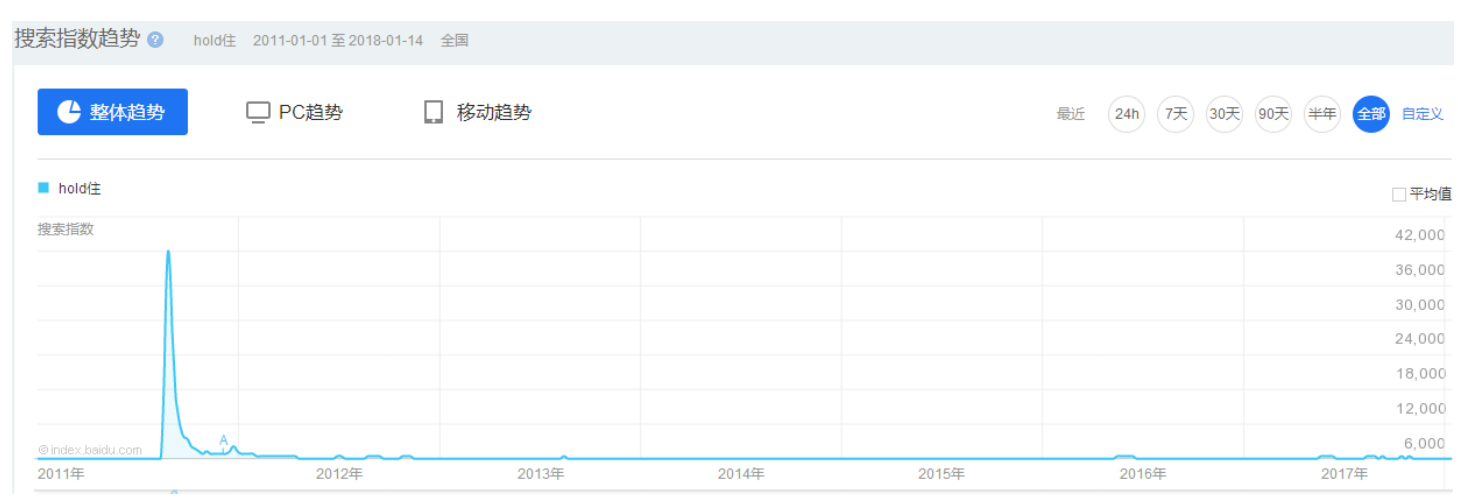

Figure 6. The diachronic using frequency of hold 住

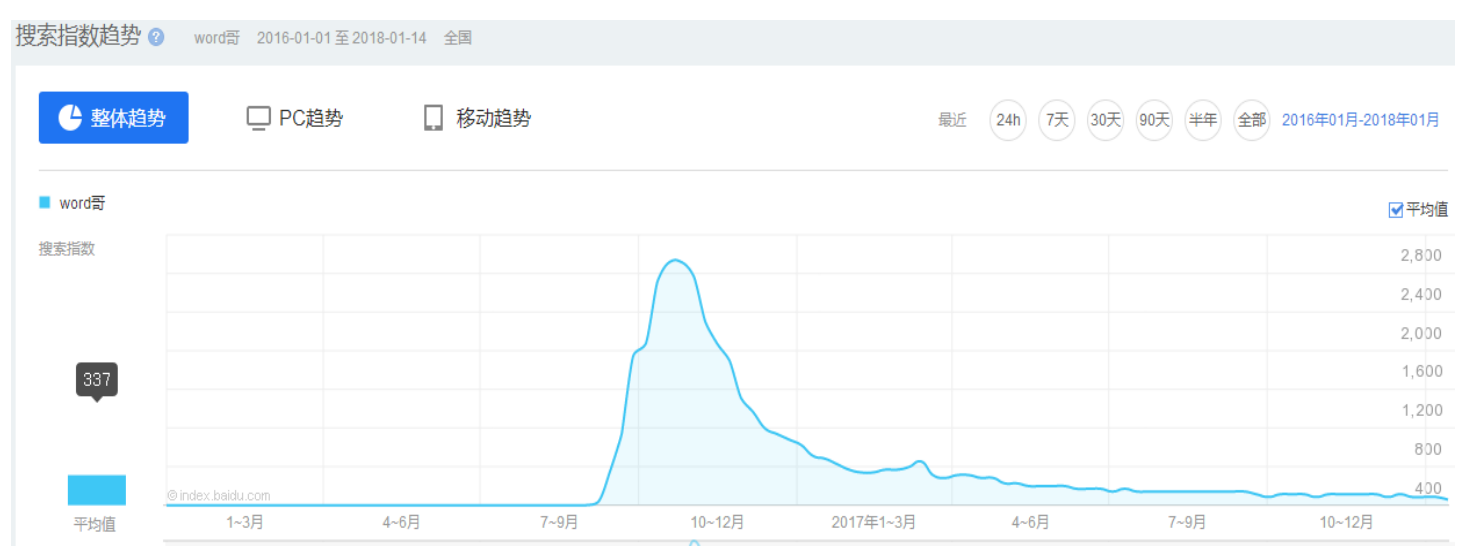

Figure 7. The diachronic using frequency of word 哥

\section{Reasons for Emergence of CEHINs}

The emerging CEHINs in recent years are resulted from the popularization of internet, the frequent cultural communications in the internet, and the further blending of English and Chinese in cyber times.

\subsection{Popularization of Internet}

The popularization of internet in China is an important reason for the emergence of Chinese-English hybridized internet neologisms. There are 0.751 billion netizens in 2017, and the popularization rate of internet is $54.3 \%$ xiii. The 'mobile phone' netizens in China account for $96.3 \%$. The fast development of internet in China makes the work, learning and communications much more convenient, which also promotes the creativity to meet the diverse individual needs of Chinese netizens. The CEHINs initially emerged in 2011, and the number is gradually increasing, with the increase of Chinese netizens. Before 2011, there are no CEHINs used in China mainland cyber world. The author can say that the CEHINs 打 call, hold 住, word 哥 are one of the products of popularization of internet in China.

\subsection{Cultural Communications}

The CEHINs are one of the products of cultural communications. According to related materials, the original concept of 
the hybridized neologisms 打 call, hold 住are respectively from Japan and Taiwan. The concept 打 call initially appeared in Japan ${ }^{\text {xiv }}$ and was introduced into Chinese cyber world, being popular among Chinese netizens: one of the top ten neologisms in 2017. The origin of hold 住 was from Taiwan in 2011 but was not hot and popular in Taiwan cyber worlds $^{\mathrm{xv}}$. It was borrowed by Chinese netizens and was quickly popular in the Chinese netizens, being the top one internet neologism of $2011^{\mathrm{xvi}}$. It can be seen that the neologisms 打 call, hold 住 are the result of netizens' communications, especially the cultural communications. If the Chinese netizens have not accepted Japanese-related 'cheer for' culture, the hybridized neologism 打 call would have not been popular in Chinese netizens. If the Chinese netizens have not watched the Taiwan TV program called '大学生了没” on 9th August, 2011 and enjoyed it, the hybridized neologism hold 住 would have not been the top one internet neologism in 2011, which continues to be used now.

\subsection{Blending of English and Chinese}

The emerging CEHINs show a special blending pattern of English and Chinese. The hybridized neologism, such as fJ call, usually consists of part of English word and part of Chinese character: there are two kinds of spelling systems (e.g, 打 is Chinese spelling system and call is English system.) and pronunciation systems ( $f 丁$ is pronounced according to Chinese pinyin, and call is pronounced according to the English pronunciation system.) within a Chinese-English hybridized internet neologism, which represents the deep interactive assimilation of Chinese and English in the lexical level in cyber times. The CEHINs are the result of interactive assimilation of English and Chinese, which is promoted by internet and the creativity of Chinese netizens. The rising popularization rate of English in China is another important factor resulting in the emergence of CEHINs. Over a decade, the English level and English popularization rate in China are rising, so that Chinese netizens who can speak or use English are increasing. It is naturally that the English-peaking Chinese netizens coin and tend to use the CEHINs during the cyber communications in China. Consequently, there are more and more CEHINs, which are popular in Chinese netizens.

It is impossible produce the CEHINs in China before 2000: one reason is that the proportion of Chinese who can speak or read English is small; another reason is that the popularization rate of internet is low, which limits the creativity of netizens and the convenience of communications.

In a word, the CEHINs not only show the characteristics of Chinese words but also show the characteristics of English words. They represent a new blending and assimilation of the two different languages in cyber times, which is driven by the Chinese netizens. They also enrich the vocabulary of Chinese English, which is regarded as a 'developing variety of English' (Xu, 2010, Xu, et al, 2017).

\subsection{Prospects for CEHINs}

Though CEHINs are emerging in recent years, the number of the neologisms is increasing and they are welcomed by Chinese netizens. Besides the three CEHINs, there are CEHINs 很 in ('fashionable'), 太 low ('low character'), 很 out ('unfashionable'), out 曼 ('behind the times'), and so on, which are used in Chinese cyberspace. One of the features of CEHINs is that most of them are within the annual top ten Chinese internet neologisms and they are welcomed by Chinese Netizens. For example, the hybridized internet neologism hold 住 is the top one Chinese internet neologism ${ }^{\mathrm{xvi}}$ in 2011, and 打 call is top one Chinese internet neologism ${ }^{\text {xviii }}$ in 2017, and word 哥, is the ninth top annual Chinese internet neologism ${ }^{\text {xix }}$ in 2016. The life span of CEHINs is much longer than the other type of Chinese internet neologisms. For instance, Xu and Tian (2017) stated that over time, most of the English lettered words in Chinese have died out. It is true that the majority of the Chinese internet neologisms could not stand the test of time, abandoned by the Chinese netizens and disappeared suddenly. However, the trend of the three neologisms 打 call, hold 住, word 哥, which are retrieved on Baidu Index, indicates that the CEHINs might stand the test of time and continue to be popular in the netizens, because of the special structures, special semantic features and the special communication effects. Seen from Figure 6, the hybridized neologism ${ }^{\text {hold }}{ }^{\text {隹 }}$ has been used more than seven years and will be continually used by Chinese netizens.

The further rising of the popularization rate of the Internet, the development of the globalization in economies and cultures, and the pervasiveness of English as a Lingua Franca in China, will accelerate the produce and use of the CEHINs. The CEHINs show and reveal the new trend of the blending of Chinese and English, the blending of cultures, and the blending of ways of thought. It is valuable to study them. The author believes that the CEHINs which stand the test of time and expand the using registers will catch the attention of scholars and official institutions. The English-Chinese lexicographers might consider the status of CEHINs, for the high using frequency and a great many users, the structure and semantic features, and the impact on the net language in China. However, whether or not the CEHINs can enter the dictionaries is an issue that time can give the answer to. 


\section{Conclusion}

The emerging CEHINs are one of products of the blending of Chinese and English on the lexical level in cyber times. They share the features of Chinese words and English words so that once they are coined, they are welcomed by the Chinese netizens in net communications, being the annual top neologisms in China. Based on Biadu Index, the author finds: (1) the structure and pronunciation of CEHINs (打 call, hold 住, word 哥) is different from the 'pure' Chinese or English internet neologisms; (2) the diffusion of them is Chinese netizen-driven, not the meme-driven; (3) the top users of them are usually aged between 30-39, while the 'pure' Chinese internet neologisms are much more frequently used by netizens aged 20-29; and (4) the popularization of internet, fast cultural communications in cyber times, and the further pervasiveness of English as a Lingua Franca in China are the reasons for the emergence and popularity of them in China. The number of CEHINs are increasing and they will have more influence on the Chinese net language, even Chinese.

\section{Acknowledgement}

This article is supported by Education Management Section in Sichuan University of Arts and Science [Grant No.: 2020JY013].

\section{References}

Bod, R., et al. (eds.) (2003). Probabilistic Linguistics. Massachuttes: MIT Press. https://doi.org/10.7551/mitpress/5582.001.0001

Liu, H., \& Lin, Y. (2018). Methodology and trends of linguistic research in the era of big data. Journal of Xinjiang Normal University(Philosophy and Social Sciences), 39(1), 72-83.

Roigmarín, A. (2016). "Blended" cyber-neologisms: new words show how our world is changing. English Today, 32(4), 2-5. https://doi.org/10.1017/S0266078416000274

Schmid, H. (2008). New words in the mind: Concept-formation and entrenchment of neologisms. Anglia-Zeitschrift für englische Philologie, 126(1), 1-36. https://doi.org/10.1515/angl.2008.002

The Lexicon of Common Words Contemporary Chinese Project Team. (2008). List of Lexicon of Common Words Contemporary Chinese (draft). Beijing: Commercial Press.

Xu, M., \& Tian, C. (2017). Is "NBA" Chinese or English? The current situation of English lettered words in Chinese. English Today, 33(4), 33-38. https://doi.org/10.1017/S0266078417000232

$\mathrm{Xu}, \mathrm{Z}$. (2010). Chinese English: Features and Implications. Hong Kong: Open University of Hong Kong Press.

Xu, Z., et al. (2017). What We Know about Chinese English: Status, Issues and Trends. In Z. Xu et al. (eds.), Researching Chinese English: the State of the Art. (Multilingual Education 22). Switzerland: Springer International Publishing AG. https://doi.org/10.1007/978-3-319-53110-6_1

Zhang, J. (2017). Internet corpora-based diachronic research on the diffusion of Shanzhai through the internet. Journal of quantitative linguistics, 24(2-3), 197-212. https://doi.org/10.1080/09296174.2016.1265800

Zhuo, J., \& Shu, H. (to appear) (2018). Chinese neologism. In R. Chu, et al. (eds.), The Routledge Handbook of Chinese Applied Linguistics. London/New York: Routledge.

\section{Copyrights}

Copyright for this article is retained by the author(s), with first publication rights granted to the journal.

This is an open-access article distributed under the terms and conditions of the Creative Commons Attribution license (http://creativecommons.org/licenses/by/4.0/).

Note:

\footnotetext{
i See http://blog.sina.com.cn/s/blog_4c0596f60102x0mx.html

ii See http://index.baidu.com/

iii See http://www.cnnic.net.cn/hlwfzyj/hlwxzbg/hlwtjbg/201708/P020170807351923262153.pdf

iv See https://baike.baidu.com/item/\%E7\%99\%BE\%E5\%BA\%A6\%E6\%8C\%87\%E6\%95\%B0/1 06226 ?fr=aladdin

v See http://index.baidu.com/Helper/?tpl=help\&word=

vi See http:// www.wordandphrase.info/frequencyList.asp

vii See https://baike.baidu.com/item/\%E7\%99\%BE\%E5\%BA\%A6\%E6\%8C\%87\%E6\%95\%B0/1
} 
$06226 ?$ fr $=$ aladdin

viii The number in the brackets are the ranks of the frequency of Chinese characters in the List of Lexicon of Common Words Contemporary Chinese (draft).

ix The number in the brackets are the ranks of the frequency of English words according to the word frequency list of 60, 000 words. See https://www.wordandphrase.info/frequencyList.asp

x See mall.cnki.net/magazine/articlesearchnew.aspx?keys=打 call

xi See http://www.cnnic.net.cn/hlwfzyj/hlwxzbg/hlwtjbg/201701/P020170123364672657408.pdf

xii See https://baike.baidu.com/item/hold\%E4\%BD\%8F/1226204

xiii See http://www.internetworldstats.com/stats.htm

xiv See https://baike.baidu.com/item/\%E6\%89\% 93call/22064311?fr=aladdin

xv See https://baike.baidu.com/item/hold\%E4\%BD\%8F/1226204?fr= aladdin

xvi See http://blog.sina.com.cn /s/blog_603d862d0102dvj3.html

xvii See http://blog.sina.com.cn/s/blog_603d862d0102dvj3.html

xviii See http://tech.sina.com.cn/roll/2017-12-20/doc-ifypxmsq8368945.shtml

xix See https://baijiahao.baidu.com/s?id=1551938069796931\&wfr=spider\&for=pc 